\title{
Survey on Robotics and Automation Technologies for Civil Infrastructure
}

\author{
Hyun Myung ${ }^{1}$, Yang Wang ${ }^{2}$, Shih-Chung Jessy Kang ${ }^{3}$, and XiaoQi Chen ${ }^{4}$ \\ ${ }^{1}$ Dept. of Civil and Environmental Engineering, KAIST, Daejeon, Republic of Korea \\ ${ }^{2}$ School of Civil and Environmental Engineering, Georgia Institute of Technology, Atlanta, GA, USA \\ ${ }^{3}$ Dept. of Civil Engineering, National Taiwan University, Taipei, Taiwan \\ ${ }^{4}$ Dept. of Mechanical Engineering, University of Canterbury, Christchurch, New Zealand
}

\begin{abstract}
Over the past several decades, substantial amounts of sensors and sensing systems have been developed for civil infrastructure systems. This special issue focuses on state-of-the-art robotics and automation technologies, including construction automation, robotics, instrumentation, monitoring, inspection, control, and rehabilitation for civil infrastructure. The issue also covers construction informatics supporting sensing, analysis and design activities needed to operate smart and sustainable civil infrastructure. Examples include robotic systems applied to civil infrastructure and equipped with various sensing technologies, such as optical sensors, laser sensors, wireless sensors, multi-sensor fusion, etc. This special issue is published in an effort to disseminate current advances of various robotics and automation technologies for civil infrastructure and built environment.
\end{abstract}

Keywords: civil infrastructure; structural health monitoring (SHM); sensing; inspection; robotics; automation

\footnotetext{
1 Associate Professor, Guest Editor, E-mail: hmyung@kaist.ac.kr

2 Associate Professor, Guest Editor, E-mail: yang.wang@ce.gatech.edu

3 Associate Professor, Guest Editor, E-mail: sckang@ ntu.edu.tw

${ }^{4}$ Professor, Guest Editor, E-mail: xiaoqi.chen@ canterbury.ac.nz

All authors have contributed equally to this work.
} 


\section{Introduction}

For several decades, various sensors and sensing systems have been developed to monitor and assess the safety condition of structures. Structural health monitoring (SHM) is an essential component in civil engineering for safety and integrity of civil structures such as buildings, bridges, power plants, off-shore structures, and tunnels. Meanwhile, advanced sensing and robotics technologies greatly facilitated construction automation of infrastructure systems. This survey paper discusses sensing and robotics technologies in SHM, inspection, and construction automation systems. These include robotic systems equipped with various sensing and sensor fusion technologies, such as optical sensors, laser sensors, accelerometers, mobile wireless sensors, etc.

Thus far, robotic development for SHM has concentrated primarily on the inspection of structures rather than the continuous monitoring of structures. This is because inspection for civil infrastructure is a time consuming and labor intensive procedure as civil infrastructure often consists of large scale engineering systems. Robots specialized for SHM applications have only recently been developed. These robots have unique locomotion systems to provide mobility in the structures to be inspected, and are equipped with various sensors such as cameras, lasers, RF (radio frequency), optical fibers, accelerometers, etc. to monitor and assess structural condition.

Compared with decades ago, modern civil engineering has the unprecedented advantages of benefiting from state-of-the-art sensing technologies. Both in research and in practice, the civil engineering field has seen more and more real-world and large-scale sensor applications. While some instrumentation systems remain cabled, others have started the revolutionary shift to miniature wireless sensing devices or even mobile sensors (i.e. small tetherless robots carrying wireless sensors). Either way, the rapidly evolving sensing technologies are providing unprecedented amounts of information about our complex civil structures operating in harsh conditions in the field. The advances in large-scale structural sensing, identification, and monitoring have been remarkable and revolutionary over the past few decades.

In Section 2, recent advances in robotics and automation for civil infrastructure are discussed, with a focus on sensing systems and robotic inspection systems. In Section 3, the papers published in this 
special issue are briefly discussed. Conclusions are given in Section 4.

\section{Recent advances in robotics and automation for civil infrastructure}

\subsection{Sensing technology and multi-sensor fusion for civil infrastructure}

An SHM system measures structural performance and operating conditions with various types of sensing devices, and evaluates safety conditions using damage diagnosis or prognosis methods (Chang, et al. 2003, Farrar, et al. 2003, Sohn, et al. 2004, Glaser, et al. 2007). Seismic structural displacement measurement using line-scan cameras has been investigated (Nayyerloo, et al. 2011). For measuring foundation pile movements, multiple displacements and motions of any structure can be determined in real time at rates over $1 \mathrm{kHz}$. A novel edge tracking algorithm is proposed that enables high-resolution measurement of large motions using relatively low-resolution, line-scan cameras.

Among many advances in SHM research, smart wireless sensors capable of embedded computing and wireless communication have attracted wide interest. The ability to incorporate sensors with wireless communication and embedded computing has motivated a flurry of research in developing wireless sensors for SHM (Straser and Kiremidjian 1998, Glaser 2004, Lynch, et al. 2004, Shinozuka, et al. 2004, Spencer, et al. 2004, Liu, et al. 2005, Wang, et al. 2005, Lynch and Loh 2006, Zhang and Li 2006, Kim, et al. 2007, Nagayama and Spencer 2007). Not only laboratory demonstrations, but also large-scale field applications of wireless SHM research have grown at an impressive rate (Lynch, et al. 2006, Zimmerman, et al. 2008, Pakzad 2010, Rice, et al. 2010). Recently, Rowe et al. (2011) presented a sensor network called Sensor Andrew for managing the large-scale infrastructure. Lin et al. (2012) developed a detection method of the damage location of the building with a wireless sensing system. In a typical application, wireless sensing nodes are associated with high-precision accelerometers for vibration measurements. Although recent development in wireless sensing devices helps reduce hardware expenses, the cost of a wireless node with a high-precision accelerometer is still at least several hundred dollars, which makes dense instrumentation still prohibitively expensive for 
large structures.

Compared with static sensor networks, mobile sensor networks offer flexible architectures with adaptive and high spatial resolutions that can provide abundant detailed information about the structure. It is anticipated that by implanting mobility into traditional sensors, mobile sensor networks will play an essential role in next revolution of sensor networks (Kahn, et al. 1999, Akyildiz, et al. 2002, LaMarca, et al. 2002). Motivated by the interest to incorporate mobility into traditional sensors, a few inspection robots have been developed for SHM. For example, a robot able to crawl on a 2D surface was developed for visually inspecting aircraft exterior; the robot used ultrasonic motors for mobility and suction cups for adherence (Backes, et al. 1997). A beam-crawler has been developed for wirelessly powering and interrogating battery-less peak-strain sensors; the crawler moves along the flange of an I-beam by wheels (Huston, et al. 2001). Based upon magnetic on-off robotic attachment devices, a magnetic walker has been developed for maneuvering on a 2D surface (Brian, et al. 2004).

More recently, a remotely-controlled model helicopter was illustrated for charging and communicating with wireless sensors (Todd, et al. 2007). Furthermore, researchers have recently developed several mobile sensing nodes (MSN) for SHM. One example prototype incorporates a flexible compliant beam between two magnet-wheeled cars (Lee, et al. 2009). As demonstrated by Guo, et al. (2009), the accelerometer can be attached onto or detached from the structural surface by bending or straightening the compliant beam of the flexure-based MSN, which also offers flexibility for transiting over concave or convex corners of a steel portal frame (Zhu, et al. 2010). The MSNs are also demonstrated to be able to navigate on a steel pedestrian bridge on Georgia Tech campus (Zhu, et al. 2012), successfully illustrating the potential of MSNs in providing high spatial resolution for sensor data collection.

\subsection{Robotic inspection for civil infrastructure}

Robotic inspection technologies have been broadly used for civil infrastructure. Chen et al. (2014) developed a lightweight bridge inspector, which uses a dual-cable suspension mechanism lifting 
camera below the bridge to capture the crack under the bridge. Su et al. (2013) developed a real-time pavement inspector, which uses a dual-light inspection method to improve the accuracy of the original image processing method. Recently, the researches utilizing UAVs in civil engineering are increasing. Many researchers use UAVs to capture aerial photos and these photos are used to build digital 3D model through image processing and digital modeler technology. Using UAVs to capture aerial photos has two advantages: low cost and high mobility. UAVs can enter the place where humans are not easy to enter.

Based on the locomotion mechanisms, inspection robots can be classified as follows. It should be noted that only recent developments are addressed and more examples can be found in Myung, et al. (2014).

1) Wheeled robots moving on horizontal surfaces: Lim et al. (2011) proposed a mobile robot equipped with a camera and a laser sensor for crack inspection of bridges. Lee and Mita (2012) proposed a mobile robot with accelerometers for evaluating structural conditions and a laser range finder (LRF) for simultaneous localization and mapping (SLAM).

2) Wall-climbing / Crawling robots: For movement on vertical walls or uneven surfaces, geckolike locomotion using directional adhesion, a vacuum-creating suction adhesion, and a climbing mechanism using adhesive materials have been developed. Teleoperated climbing inspection robots have been developed by Bao et al. (2012) and a bio-inspired miniature wallclimbing caterpillar robot has been developed by Zhang et al. (2010). A micro aerial vehicle type wall-climbing robot was also developed (Shin, et al. 2013), which used thrust forces induced by the four rotors not only to fly but also to stick on the wall.

3) Snake-like robots: These robots are suitable for inspecting narrow spaces and rough terrain that are hard to navigate. Tokyo Tech's Hirose-Fukushima Lab has developed softly moving mechanical snakes (Mori and Hirose 2006).

4) Modular robots: It is possible to build a robot capable of going through rough terrain and uneven surfaces by cascading multiple small modular robots. A camera-installed cable inspection robot composed of two modules has been developed by $\mathrm{Xu}$ et al. (2011). The 
proposed robot stably climbs random inclined cables and lands smoothly by using a gas damper with a slider-crank mechanism.

5) Aerial vehicles: Robots in the form of quadrotors, helicopters, and airships can freely fly through and inspect structures. Quadrotors and miniature helicopters have been extensively tested. Most of the current prototypes have limited payload capacity and thus cannot carry many sensors (Pratt, et al. 2008, Shin, et al. 2013).

6) Underwater vehicles: Submersible robots have been developed to inspect underwater structures. Several prototypes have been designed to inspect oil storage tanks (Abdulla, et al. 2010) and pipelines (Conte, et al. 1996, Bodenmann, et al. 2009).

Various sensors, such as optical sensors, lasers, and their combinations have been incorporated for robotic structural inspection purposes (Myung, et al. 2011, Jeon, et al. 2011, Jeon, et al. 2012).

\section{Summary of this special issue}

The following papers included in this special issue on "Robotics and Automation for Civil Infrastructure" properly address these current topics of interest and are briefly summarized here.

\subsection{Structural health monitoring using robotic technologies}

Adhikari et al., in their paper, "Automated condition assessment of concrete bridges with digital imaging", present an approach of automating the prediction of condition rating for bridges based on digital image analysis. The proposed methodology encompasses image acquisition, development of 3D visualization model, image processing, and condition rating model. Under this method, scaling defect in concrete bridge components is considered as a candidate defect and the guidelines in the Ontario Structure Inspection Manual (OSIM) have been adopted for developing and testing the proposed method. The automated algorithms for scaling depth prediction and mapping of condition ratings are 
based on training of back propagation neural networks.

Jeon et al., in their paper, "Vision-based remote 6-DOF structural displacement monitoring system using a unique marker," propose a vision-based displacement measurement system with a uniquely designed marker to monitor the 6-DOF structural displacement with high accuracy. The system is composed of a uniquely designed marker and a camera with a zooming capability, and relative translational and rotational displacement between the marker and the camera is estimated by finding a homography transformation. The novel marker is designed to make the system robust to measurement noise based on a sensitivity analysis of the conventional marker and it has been verified through simulations and experiments.

\subsection{Sensing technology and multi-sensor fusion for civil infrastructure}

Wang et al., in their paper, "Fatigue performance monitoring of full-scale PPC beams by using the FBG sensors," conducted an experimental study of fatigue performance monitoring of partially prestressed concrete (PPC) structure by using Fiber Bragg Grating (FBG) sensors. The strain is determined by measuring the wavelength shift and the temperature shift. The experiments are conducted on full-scale post-tensioned PPC beams under fatigue loading. The performance of both the FBG and resistance strain gauge (RSG) sensors is evaluated and compared. The experimental results show that FBG sensors have relatively good resistance to fatigue loading compared with RSG sensors, and hence are suitable for long-term health monitoring of the tensile reinforcement in PPC structures.

Gomathi et al., in their paper, "Hand arm vibration measurement using micro-accelerometer in different brick structures," present a MEMS capacitive area changing micro-accelerometer for safety monitoring of construction workers operating power tools. The accelerometer measures the hand arm vibrations experienced by workers and warn them when breaching the nominal values specified by ISO safety standards. The MEMS accelerometer exhibits superior performance in the low frequency range combined with linearity, low noise performance and high sensitivity. Using different types of blocks as operating subject, comparative experiments are carried out with a conventional accelerometer to study the advantages and disadvantages of the MEMS accelerometer. 
Bai et al., in their paper, "Crack location in beams by data fusion of fractal dimension features of laser-measured operating deflection shapes," propose a method for beam crack localization using data fusion of fractal dimension features of operating deflection shapes (ODSs). Data fusion of Katz's fractal dimension (KFD) curves of various ODSs is used collectively to create a fractal damage feature, i.e. the overall KFD curve. The method is demonstrated in numerical and experimental cases of cantilever beams with single/multiple cracks. For the experimental case, a scanning laser vibrometer is used to acquire high-resolution ODSs.

Luo et al., in their paper, "Development of a dynamic sensing system for civil revolving structures and its field tests in a large revolving auditorium," discuss a wireless dynamic sensing system for a large revolving structure (RS). Revolving structures are a unique structural form which is seen in innovative architecture design. Such structures revolve around themselves or along a certain track. Safety design and health monitoring of RS are critical, but have not attracted much attention from researchers. The proposed system consists of one sink and multiple vibrating wire sensors (VWS) nodes for strain measurement. It dynamically assigns the addresses of sensor nodes when RS position changes. The proposed method is validated with field tests on a rotating auditorium. The work demonstrates effective implementation of WSN for civil structures.

Chiu et al., in their paper, "Integration of in-situ load experiments and numerical modeling in a long-term bridge monitoring system on a newly-constructed widened section of freeway in Taiwan," propose a real-time bridge monitoring system. The research considered several results of the bridge experiment and established the structural models of the bridge. The research provides the simulation approach for real bridges and can be utilized for bridge management.

\subsection{Robotic inspection for civil infrastructure}

Lee et al., in their paper, "Two-module robotic pipe inspection system with EMATs," introduce a two-module robotic pipe inspection system with ultrasonic NDE device to evaluate the integrity of pipe structures. The proposed robotic platform has high mobility. The two module mobile robot 
platform overcomes pipe obstacle structures such as elbow, or T-branch joints by cooperative maneuvers. Also, it can climb up the straight pipeline at a fast speed due to the wheel driven mechanism. For inspection of pipe structures, SH-waves generated by EMAT are applied with additional signal processing methods.

Liu et al., in their paper, "A review of rotorcraft Unmanned Aerial Vehicle (UAV) developments and applications in civil engineering," extensively overview the unmanned aerial vehicle applied to civil structures including its hardware (frame structure, electromechanics, flight controller and telemetry control system), software and related technologies (control, power management, navigation and image processing). Their paper also analyzes the opportunities and challenges of applying UAV in civil engineering applications including seismic risk assessment, transportation, disaster response, construction management, surveying and mapping, and flood monitoring and assessment.

\section{Conclusions}

Although sensor technologies have revolutionized over the past few decades, critical challenges for civil infrastructure applications still exist. Besides measurement accuracy, the multi-faceted issues includes sensor miniaturization, low cost, support of wireless communication (or even further mobility for mobile sensors), sensor smartness (with embedded computing), long-term powering, durability, etc. Nevertheless, it is expected that in the coming decades, civil infrastructure systems will continue benefiting from rapidly evolving sensing technologies. On the other hand, for civil engineering researchers, sensor data inundation is becoming more and more of a reality. There is a growing demand for data fusion techniques that are able to scour through megabytes and gigabytes of data, collected by tens or hundreds of various types of sensors, and convert the large volume of data into information that is meaningful and actionable for infrastructure stakeholders. There will be increasing integration of sensor technologies, mobile robots, wireless communication for resource monitoring, management, and automated construction. History will see that advances in both sensor technologies and sensor data processing are among the most remarkable revolutions for civil engineering of our era. 
Furthermore, using robotics to assist civil infrastructure inspection procedures can save significant time, labor, and money. Technological advances are providing unprecedented opportunities of adopting robots for SHM and inspection applications. Carrying a variety of sensors, the robots with unique locomotion mechanisms can provide autonomous mobility while inspecting structures. Examples for different locomotion mechanism include wheeled robots moving on horizontal surfaces, wall-climbing and crawling robots, snake-like robots, modular robots, aerial vehicles, underwater vehicles, etc. Inspection robots have been applied to various target structures such as aircrafts, bridges, power lines, pipelines, ducts, tubes, sewages, etc. Although robots specialized for SHM applications have only recently been developed, their use is expected to largely increase in the near future.

\section{Acknowledgments}

The Guest Editors of this special issue wish to acknowledge the valuable support of the Prof. Chung-Bang Yun, Editor-in-Chief, during all stages of the effort. Special thanks to Ms. Hye Jeong Lee of Techno Press for her efforts. Guest Editor Y. Wang would like to thank the support from the U.S. National Science Foundation (CMMI-1150700). Any opinions, findings, and conclusions or recommendations expressed in this publication are those of the authors and do not necessarily reflect the view of the sponsor.

\section{References}

Abdulla, A.S.A., Khalil, A.M.A., Shehhi, A., Karki, H. and Fok, S.C. (2010), "Localization of a submersible mobile inspection platform in an oil storage tank", Proc. 7th International Symposium on Mechatronics and its Applications (ISMA), 1 - 6.

Bodenmann, A., Thornton, B., Ura, T. and Painumgal, U.V. (2009), "Visual mapping of internal pipe walls using sparse features for application on board Autonomous Underwater Vehicles", Proc. of OCEANS 2009 - EUROPE, $1-8$.

Chen, Y.C., Yang, C.E. and Kang, S.C. (2014), “A Lightweight Bridge Inspection System Using a 
Dual-cable Suspension Mechanism", Automation in Construction. Accepted in April.

Conte, G., Zanoli, S., Perdon, A.M., Tascini, G. and Zingaretti, P. (1996), “Automatic analysis of visual data in submarine pipeline inspection", Proc. of MTS/IEEE.OCEANS '96, 3, 1213 - 1219.

Akyildiz, I.F., Su, W., Sankarasubramaniam, Y. and Cayirci, E. (2002), “A survey on sensor networks”, IEEE Commun. Mag., 40(8), 102-114.

Backes, P.G., Bar-Cohen, Y. and Joffe, B. (1997), "The multifunction automated crawling system (MACS)", Proc. of the 1997 IEEE International Conference on Robotics and Automation, Albuquerque, New Mexico.

Bao, Y., Wu, F., Zhu, X., Zhang, X. and Li, H. (2012), “A mobile wireless sensor-based structural health monitoring technique", in Proc. of workshop on civil structural health monitoring, Berlin.

Brian, E., Jon, M., Dryver, R.H. and Phil, B. (2004), "Robotic systems for homeland security", Proc. of SPIE, Nondestructive Detection and Measurement for Homeland Security II, San Diego, CA.

Chang, P.C., Flatau, A. and Liu, S.C. (2003), "Review paper: health monitoring of civil infrastructure", Struct. Health Monit., 2(3), 257-267.

Farrar, C.R., Sohn, H., Hemez, F.M., Anderson, M.C., Bement, M.T., Cornwell, P.J., Doebling, S.W., Schultze, J.F., Lieven, N. and Robertson, A.N. (2003), Damage Prognosis: Current Status and Future Needs. LA-14051-MS, Los Alamos National Laboratory, Los Alamos, NM.

Glaser, S.D. (2004), "Some real-world applications of wireless sensor nodes", Proc. of SPIE, Smart Structures and Materials 2004: Sensors and Smart Structures Technologies for Civil, Mechanical, and Aerospace Systems, San Diego, CA.

Glaser, S.D., Li, H., Wang, M.L., Ou, J. and Lynch, J.P. (2007), "Sensor technology innovation for the advancement of structural health monitoring: a strategic program of US-China research for the next decade", Smart Struct. Syst., 3(2), 221-244.

Guo, J., Lee, K.-M., Zhu, D. and Wang, Y. (2009), "A flexonic magnetic car for ferro-structural health monitoring", Proc. of 2009 ASME Dynamic Systems and Control Conference, Hollywood, CA, USA.

Huston, D.R., Esser, B., Gaida, G., Arms, S.W. and Townsend, C.P. (2001), "Wireless inspection of 
structures aided by robots", Proc. of SPIE, Health Monitoring and Management of Civil Infrastructure Systems, Newport Beach, CA.

Jeon, H., Shin, J.-U., and Myung, H. (2012), "Incremental displacement estimation of structures using paired structured light," Smart Structures and Systems, 9(3), 273-286.

Jeon, H., Bang, Y. and Myung, H. (2011), “A paired visual servoing system for 6-DOF displacement measurement of structures", Smart Mate. Struct, 20, 045019.

Kahn, J.M., Katz, R.H. and Pister, K.S.J. (1999), "Next century challenges: mobile networking for "Smart Dust"”, Proc. of the 5th Annual ACM/IEEE International Conference on Mobile Computing and Networking, Seattle, Washington.

Kim, S., Pakzad, S., Culler, D., Demmel, J., Fenves, G., Glaser, S. and Turon, M. (2007), "Health monitoring of civil infrastructures using wireless sensor networks", Proc. of the 6th International Conference on Information Processing in Sensor Networks (IPSN '07), Cambridge, MA.

LaMarca, A., Brunette, W., Koizumi, D., Lease, M., Sigurdsson, S.B., Sikorski, K., Fox, D. and Borriello, G. (2002), "Making sensor networks practical with robots", Proc. of the First International Conference on Pervasive Computing, Zurich, Switzerland.

Lee, K.-M., Wang, Y., Zhu, D., Guo, J. and Yi, X. (2009), "Flexure-based mechatronic mobile sensors for structure damage detection", Proc. of the 7th International Workshop on Structural Health Monitoring, Stanford, CA, USA.

Lee, N. and Mita, A. (2012), "Sensor agent robot with servo-accelerometer for structural health monitoring," Proc. of SPIE, San Diego.

Lim, R. S., La, H. M., Shan, Z. and Sheng, W. (2011), “Developing a crack inspection robot for bridge maintenance," Proc. of IEEE Int. Conf. Robot. Autom., Shanghai.

Lin, T.K., Hung, S.L. and Huang C.S. (2012), "Detection of Damage Location Using a Novel Substructure-Based Frequency Response Function Approach with a Wireless Sensing System,” International Journal of Structural Stability and Dynamics, 12(4), 1250029.

Liu, L., Yuan, F.G. and Zhang, F. (2005), "Development of wireless smart sensor for structural health monitoring", Proc. of SPIE, Smart Structures and Materials 2005: Sensors and Smart Structures Technologies for Civil, Mechanical, and Aerospace Systems, San Diego, CA. 
Lynch, J.P., Sundararajan, A., Law, K.H., Kiremidjian, A.S. and Carryer, E. (2004), "Embedding damage detection algorithms in a wireless sensing unit for operational power efficiency", Smart Mater. Struct., 13(4), 800-810.

Lynch, J.P., Wang, Y., Loh, K.J., Yi, J.-H. and Yun, C.-B. (2006), "Performance monitoring of the Geumdang Bridge using a dense network of high-resolution wireless sensors", Smart Mater. Struct., 15(6), 1561-1575.

Lynch, J.P. and Loh, K.J. (2006), "A summary review of wireless sensors and sensor networks for structural health monitoring”, Shock Vib. Dig., 38(2), 91-128.

Mori, M. and Hirose, S. (2006), "Locomotion of 3D Snake-Like Robots; Shifting and Rolling Control of Active Cord Mechanism ACM-R3”, Journal of Robotics and Mechatronics, 18, 521-528.

Myung, H., Lee, S. and Lee, B.-J. (2011), "Paired Structured Light for Structural Health Monitoring Robot System”, Int'l Journal of SHM (Structural Health Monitoring), 10(1), 49-64.

Myung, H., Jeon, H., Bang, Y.-S., and Wang, Y. (2014), "Robotic sensing for assessing and monitoring civil infrastructures”, Sensor Technologies for Civil Infrastructures, Wang, M.L., Lynch, J.P., and Sohn, H. (eds.), 410-445, Woodhead Publishing Limited

Nagayama, T. and Spencer, B.F., Jr. (2007), Structural Health Monitoring using Smart Sensors. NSEL001, Newmark Structural Engineering Laboratory, University of Illinois at Urbana-Champaign, Urbana, IL.

Nayyerloo, M., Chase, J.G., Millane, A., Muller, C., Malherbe, A., Chen, X.Q., MacRae, G.A (2011), "Seismic structural displacement measurement using a line-scan camera: camera-pattern calibration and experimental validation”, J Civil Structure Health Monitoring, 1, 113-124.

Pakzad, S.N. (2010), "Development and deployment of large scale wireless sensor network on a longspan bridge”, Smart Struct. Syst., 6(5), 525-544.

Pratt, K. S., Murphy, R. R., Burke, J. L., Craighead, J., Griffin, C. and Stover, S. (2008), "Use of Tethered Small Unmanned Aerial System at Berkman Plaza II Collapse," Proc. of IEEE International Workshop on Safety, Security and Rescue Robotics (SSRR 2008), 134 - 139.

Rice, J.A., Mechitov, K., Sim, S.-H., Nagayama, T., Jang, S., Kim, R., B. F. Spencer, J., Agha, G. and Fujino, Y. (2010), "Flexible smart sensor framework for autonomous structural health 
monitoring”, Smart Struct. Syst., 6(5), 423-438.

Rowe, A., Berges, M.E., Bhatia, G., Goldman, E., Rajkumar, R., Garrett, J.H., Moura, J.M.F. and Soibelman, L. (2011), "Sensor Andrew: Large-scale Campus-wide Sensing and Actuation”, IBM Journal of Research and Development, 55(1.2), 6:1-6:14.

Shin, J.-U., Kim, D., Kim, J.-H., and Myung, H. (2013), "Micro aerial vehicle type wall-climbing robot mechanism," Proc. IEEE RO-MAN(IEEE International Symposium on Robot and Human Interactive Communication), 722-725, Gyeongju, Korea.

Shinozuka, M., Feng, M.Q., Chou, P., Chen, Y. and Park, C. (2004), "MEMS-based wireless real-time health monitoring of bridges", Proc. of the 3rd International Conference on Earthquake Engineering, Nanjing, China.

Sohn, H., Farrar, C.R., Hemez, F.M., Shunk, D.D., Stinemates, D.W. and Nadler, B.R. (2004), A Review of Structural Health Monitoring Literature: 1996-2001. LA-13976-MS, Los Alamos National Laboratory, Los Alamos, NM.

Spencer, B.F., Jr., Ruiz-Sandoval, M.E. and Kurata, N. (2004), "Smart sensing technology: opportunities and challenges", Struct. Control Hlth. Monit., 11(4), 349-368.

Straser, E.G. and Kiremidjian, A.S. (1998), "A Modular, Wireless Damage Monitoring System for Structures", Technical Report 128, John A. Blume Earthquake Eng. Ctr., Stanford University, Stanford, CA.

Su, Y.S., Kang, S.C., Chang , J.R. and Hsieh, S.H. (2013), "Dual-Light Inspection Method for Automatic Pavement Surveys”, Journal of Computing in Civil Engineering, 27(5), 534-543.

Todd, M., Mascarenas, D., Flynn, E., Rosing, T., Lee, B., Musiani, D., Dasgupta, S., Kpotufe, S., Hsu, D., Gupta, R., Park, G., Overly, T., Nothnagel, M. and Farrar, C. (2007), "A different approach to sensor networking for SHM: remote powering and interrogation with unmanned aerial vehicles", Proc. of the 6th International Workshop on Structural Health Monitoring (IWSHM), Stanford, CA.

Wang, Y., Lynch, J.P. and Law, K.H. (2005), "Wireless structural sensors using reliable communication protocols for data acquisition and interrogation", Proc. of the 23rd International Modal Analysis Conference (IMAC XXIII), Orlando, FL.

Xu, F. Y., Wang, X. S. and Wang, L. (2011), “Cable inspection robot for cable-stayed bridges: Design, 
analysis, and application," J. Field Robot., 28(3), 441-459.

Zhang, H., Wang, W., Gonzalez-Gomez, J. and Zhang, J. (2010), “A Bio-Inspired Small-Sized WallClimbing Caterpillar Robot", Mechatronic Systems Applications, Paola A M D D and Cicirelli G (eds).

Zhang, Y. and Li, J. (2006), "Wavelet-based vibration sensor data compression technique for civil Infrastructure condition monitoring”, J. Comput. Civil Eng., 20(6), 390-399.

Zhu, D., Yi, X., Wang, Y., Lee, K.-M. and Guo, J. (2010), “A mobile sensing system for structural health monitoring: design and validation", Smart Mater. Struct., 19(5), 055011.

Zhu, D., Guo, J., Cho, C., Wang, Y. and Lee, K.M. (2012), "Wireless mobile sensor network for the system identification of a space frame bridge", IEEE-ASME T. Mech., 17(3), 499-507.

Zimmerman, A.T., Shiraishi, M., Swartz, R.A. and Lynch, J.P. (2008), “Automated modal parameter estimation by parallel processing within wireless monitoring systems", J. Infrastruct. Syst., 14(1), $102-113$. 